\title{
A Collaborative Biomedical Image-Mining Framework along with Image Annotation
}

\author{
Kamalpreet Kaur \\ Student \\ Sri guru granth sahib world university \\ Fatehgarh Sahib, Punjab
}

\begin{abstract}
Skin is the largest organ in our body. Cancer is a group of diseases characterized by uncontrolled growth and spread of abnormal cells. If the abnormal cell is not controlled, it can result in death. There are two types of skin cancer: malignant melanoma of the skin, and non-melanoma skin cancer (NMSC). Malignant melanoma is the less common but most serious type of skin cancer. In this paper survey how to detect skin cancer in efficient manner and his detail what kind of skin cancer it is.??
\end{abstract}

\section{General Terms}

Classification, image microscopy, image mining, intelligent planning, skin cancer, GLCM (Gray Level Co-occurrence matrix)

\section{INTRODUCTION}

Malignant melanoma, the most aggressive form of skin cancer, is one of the most dangerous increasing cancers in the world, with an estimated 76,690 people being diagnosed with melanoma and 9480 people dying of melanoma in the United States in 2013 [1]. Many dermatologists [2, 3] use clinical ABCD which means Asymmetry, Border, Color, Differential structures; Menzies'method; 7- point checklist and pattern analysis methods to diagnose and classify the lesions. In particular, it is very difficult to distinguish among lesions and even experienced dermatologists [4] have a diagnostic accuracy below $85 \%$.

Due to the increase in incidence rates, it is costly for dermatologists to manually screen every patient for melanoma. Therefore, there is a need for a system to automatically assess the risk of melanoma from photographs of a patient's skin lesion. Early work on automated systems to assess the risk of melanoma used dermoscopy images [5]-[6]. The photographs are segmented to identify the lesion area, features are extracted from the lesion, and the lesion is classified in terms of risk of melanoma. The problem is that illumination from skin surface reflectance impacts all three of those steps.

For example, in Fig.1, illumination changes across the photographs horizontally or vertically. As a result, healthy skin areas obstructed by shadows appear similar in color as the skin lesion, which results in misclassification of those areas. Illumination correction is an important preprocessing step for skin lesion photographs prior to segmentation and classification algorithms. Many illumination correction algorithms exist, but they are not designed specifically for skin lesion photographs. Common algorithms correct for illumination based on histogram equalization or the illumination-reflectance model. Histogram equalization adjusts the distribution of pixel intensities and minimizes illumination variation on a global scale.

\author{
Ada \\ Asst.professor \\ Sri guru granth sahib world university \\ Fatehgarh Sahib, Punjab
}

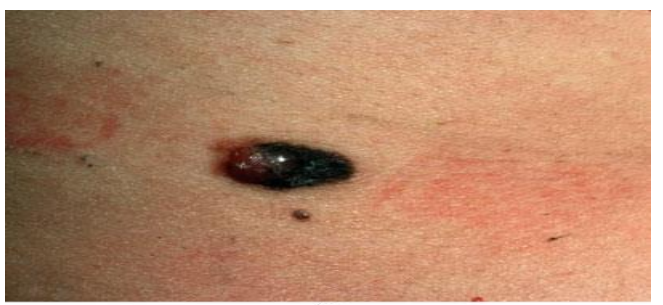

(a)

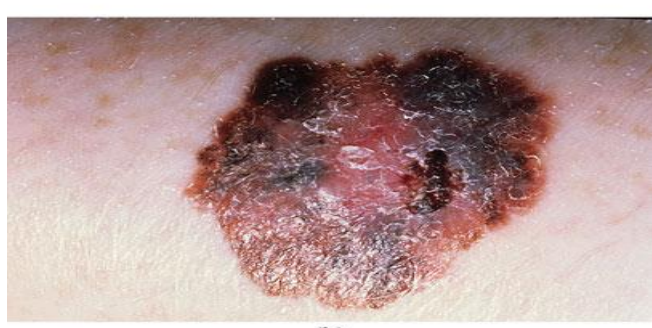

(b)

Fig 1: Examples of illumination variation in skin lesion images. In image (a), the illumination variation changes horizontally, while in image (b), it changes vertically. [7]

\section{METHODS AND TECHNIQUESIN VARIOUS STEPS OF SKIN CANCER}

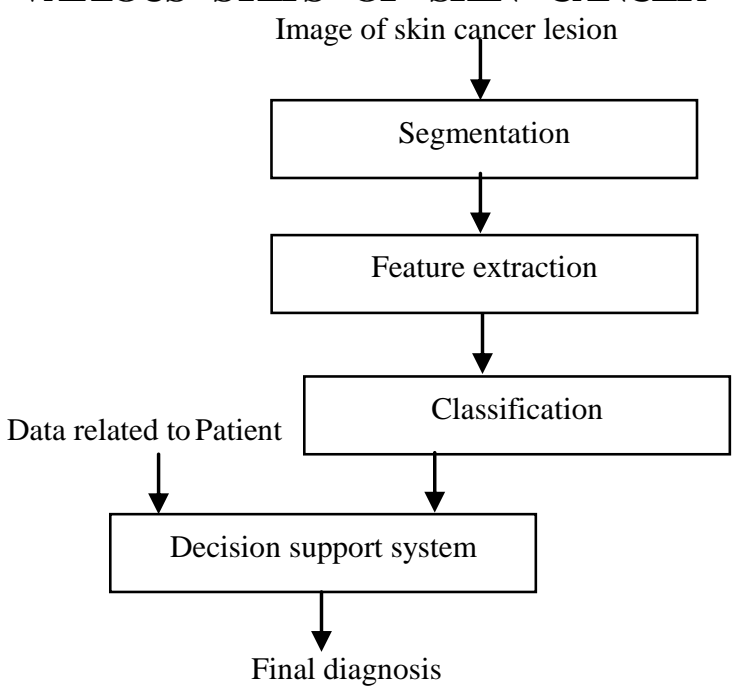

Fig 2: Overall workflow of the automatic system for melanoma screening [17]

Multistage Illumination Modeling (MSIM) Algorithm: MSIM has three stages first, an initial estimate of map is determined using a nonparametric modeling approach based on Monte Carlo sampling. Second, the final estimated illumination map is found using parametric modeling, using the nonparametric map as a prior. Finally, the illumination variation is removed. The proposed MSIM algorithm extends significantly upon the preliminary results that were presented 
in [8] through the introduction of an accelerated nonparametric modeling framework in the first stage, and a robust parametric modeling framework in the second stage for improved illumination estimation.

It is necessary to derive the underlying illumination model in the skin lesion photographs. In order to derive this model, assumptions are made about the photographs. It is assumed that the photograph is taken in a controlled environment in a doctor's office using a standard camera. The lesion in the photograph is assumed to be illuminated from a single source of white light. Due to these assumptions, only the $\mathrm{V}$ (value) channel from the HSV color space [9] is used for illumination correction. The other channels, hue and saturation, are not corrected because the light source is only white light. Furthermore, the skin lesion is found in the center of the photograph.
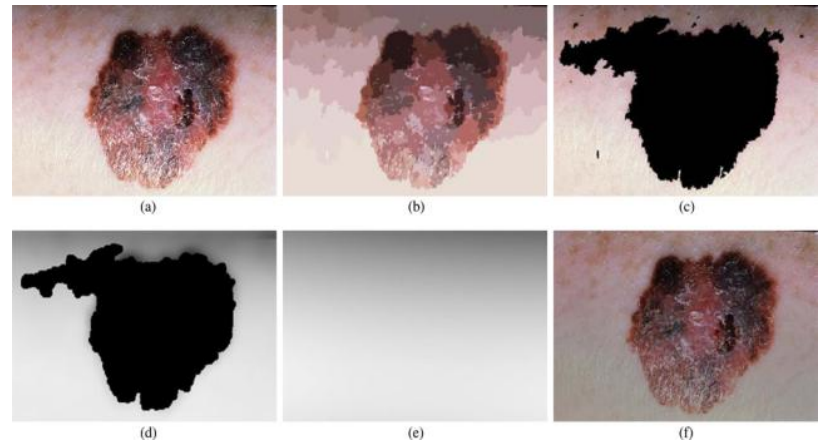

Fig 3: Methodology to calculate (a) original photograph of a skin lesion; (b) segmentation map; (c) pixels black in color are not classified as normal skin;(d) initial illumination map estimated via nonparametric modeling using Monte Carlo sampling; (e) final illumination map determined by using (d) as a prior to the parametric surface model; (f) photograph corrected for illumination variation using the MSIM algorithm.[26 ]

\section{IMAGE SEGMENTATION}

Segmentation refers to the partitioning of an image into disjoint regions that are homogeneous with respect to a chosen property such as luminance, color, texture, etc. [10]. Segmentation methods can be roughly classified into the following categories:

- Histogram thresholding

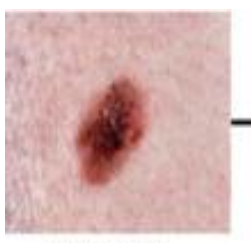

Input image

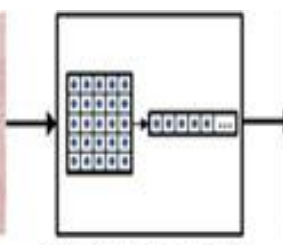

Texture representation

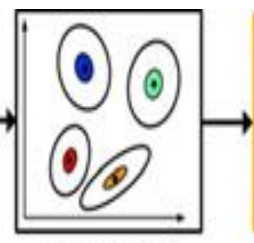

Learn texture distributions
- $\quad$ Clustering

- $\quad$ Edge-based

- $\quad$ Region-based

- $\quad$ Soft computing

Most segmentation algorithms for dermatological or photographs images use color information, either a single channel or three color channels, to find the lesion. Another approach to find skin lesions is to incorporate textural information, because normal skin and effected skin have different textures. Textures include smoothness, roughness, or the presence of bumps or other deformations and are visible by variation in pixel intensities in an area [11].

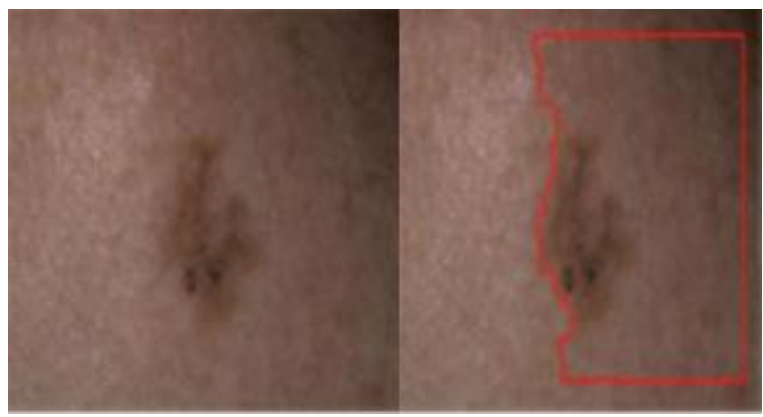

Fig 3: (a) Typical input image. (b)Erroneous segmentation results (red contour) caused by uneven background illumination. [27]

Segmentation algorithm based on texture distinctiveness (TD) to locate skin lesions in photographs. TD algorithm is referred to as the TD lesion segmentation (TDLS) algorithm. The main purposes are the introduction of a joint statistical TD metric and a texture-based region classification algorithm. TD captures the dissimilarity between learned representative texture distributions. A TD metric is calculated to measure the dissimilarity of a texture distribution from all other texture distributions. Second, the TD metric is used to classify regions in the image as part of the skin class or lesion class.

Sparse texture algorithms use sparse texture models for segmentation or classification of images with different texture and patterns. Sparse texture models find a small number of texture representations, such as texture patterns, to characterize an entire image [12].

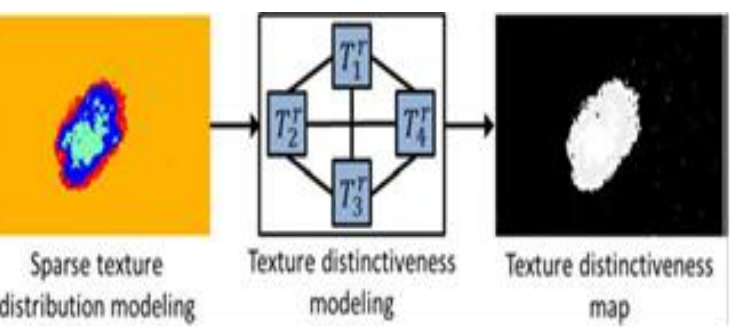

Fig 4: Algorithm flowchart displaying the steps to learn the representative texture distributions and calculate the TD metric [28]

\section{FEATURE EXTRACTION}

We extract a set of image features between benign and malignant skin lesions by using $\mathrm{ABCD}$ rule [18].

- Asymmetry

- Border

- Color

\section{- Differential Structures}

Texture Feature Extraction using GLCM

Texture features of skin cancer images are used to check the accuracy and efficiency of our proposed approach. The following features are extracted from GLCM: autocorrelation, Contrast, Energy, Entropy, homogeneity [19].Among multiscale feature extraction algorithms, the most popular are discrete wavelet transform (DWT) [20, 21], Gabor wavelets 
(GWs) [22], local binary pattern (LBP) [23], and the Steerablc pyramid transform (SPT) [24, 25]. These approaches have been shown to be very useful in capturing texture characteristics with high discriminatory power.

\section{CLASSIFICATION}

After extracting the set of features next step is classification. Boosting algorithms [29] are a set of nonparametric metalearning algorithms, provide optimal classification results. The advantages of using adaptive boosting (AdaBoost) algorithm over other machine learning algorithm are its computational efficiency, better robustness and no regressions. In medical image analysis SVM classifiers have been widely used, but as AdaBoost can choose good informative features from potentially very large feature data sets [30]. However the major problem of boosting is that, it does not provide multiple class- label outputs with maxmargin without class-correlation. Now a day boosting algorithms are extended to multi-class outputs, which are based on AdaBoost.MH [31].

\section{CONCLUSION}

In this paper, the basic concepts of skin cancer have been discussed. Then, a survey of different methods and techniques for detection of skin lesions has been done in this paper. For personal identification system, skin lesions recognition used enormous algorithms for extraction and classification purposes. It can be easily concluded that the proposed system of skin cancer is very useful for detection of skin cancers of multiple classes with a good accuracy.

\section{REFERENCES}

[1] N. Howlader, A. M. Noone, M. Krapcho, J. Garshell, N. Neyman, S. Altekruse, C. L. Kosary, M. Yu, J. Ruhl, Z. Tatalovich, H. Cho, A. Mariotto, D. R. Lewis, H. S. Chen, E.J. Feuer, and K. A. Cronin, "SEER cancer statistics review, 1975-2010," Nat. Cancer Inst., Bethesda, MD,USA, Tech. Rep., 2013

[2] R.H.Johr, Dermoscopy: alternative melanocytic algorithms-the $\mathrm{ABCD}$ rule of dermatoscopy ,menzies scoring method, and7-pointchecklist, Clinics in Dermatology 20(3)(2002)240-247.

[3] H.Blum, Luedtke, U.Ellwanger, etal.,Digital image analysis for diagnosis of cutaneous melanoma. Development of a highly effective computer algorithm based on analysis of 837melanocyticlesions, BritishJournalofDermatology 151 (5)(2004)1029-1038.

[4] G.Argenziano,H.P.Soyer,S.Chimenti,R.Talamini,R.Coro na,F.Sera,etal.,Dermoscopy of pigmented skin lesions :results of a consensusmeeting via the Internet,Journal of the American Academy ofDermatology48(5)(2003)679693.

[5] M. E. Celebi, H. A. Kingravi, B. Uddin, H. Iyatomi, Y. A. Aslandogan, W. V. Stoecker, and R. H. Moss, "A methodological approach to the classification of dermoscopy images," Comput. Med. Imag. Graph., vol. 31 , no. 6, pp. 362-373, 2007.

[6] H. Ganster, A. Pinz, R. R"uhrer, E. Wildling, M. Binder, and H. Kittler,"Automated melanoma recognition," IEEE Trans. Med. Imag., vol. 20, no. 3, pp. 233-239, Mar. 2001.

[7] S. Shan, W. Gao, B. Cao, and D. Zhao, "Illumination normalization for robust face recognition against varying lighting conditions," in Proc. IEEE Int. Workshop Anal. Model. Faces Gesture, Oct. 2003, pp. 157-164.

[8] J. Glaister, A. Wong, and D. A. Clausi, "Illumination correction in dermatological photographs using multistage illumination modeling for skin lesion analysis," in Proc. EEE 34th Annu. Int. Conf. IEng. Med. Biol. Soc., Aug./Sep. 2012, pp. 102-105.

[9] A. Smith, "Color gamut transform pairs," ACM SIGGRAPH Comput. Graph., vol. 12, no. 3, pp. 12-19, 1978.

[10] Sonka M, Hlavac V, Boyle R. Image processing, analysis, and machine vision. Cengage-Engineering 2007.

[11] M. Anantha, R. H. Moss, and W. V. Stoecker, "Detection of pigment network in dermatoscopy images using texture analysis," Comput. Med. Imag. Graph., vol. 28, no. 5, pp. 225-234, 2004.

[12] G. Peyre, "Sparsemodeling of textures," J.Math. Imag. Vis., vol. 34, no. 1, pp. 17-31, 2009.

[13] T. Glatard, J. Montagnat, and X. Pennec, "Grid-enabled workflows for data intensive medical applications," in Proc. 18th IEEE Symp. Computer- Based Med. Syst., Jun. 2005, pp. 537-542.

[14] D. Kreftin, M. Vossberg, A. Hoheisel, and T. Tolxdorff, "Simplified implementation of medical image processing algorithms into a grid using a workflow management system,” J. Future Gener. Comput. Syst., vol. 26, no. 4, pp. 681-684, Apr. 2010.

[15] C. Botha, "DeVIDE - the Delft visualization and image processing development environment," Technical University of Delft, Delft, The Netherlands, Tech. Rep. http://graphics.tudelft.nl, May 2005.

[16] S. Koulouzis, E. Zudilova-Seinstra, and A. Belloum, "Data transport between visualization web services for medical image analysis," Procedia Comput. Sci., vol. 1, no. 1, pp. 1727-1736, May 2010.

[17] José Fernández Alcón, C alina Ciuhu, Warner ten Kate, Adrienne Heinrich, Natallia Uzunbajakava, Gertruud Krekels, Denny Siem, and Gerard de Haan" Automatic Imaging System With Decision Support for Inspection of Pigmented Skin Lesions and Melanoma Diagnosis" IEEE journal of selected topics in signal processing, vol. 3, no. 1, february 2009

[18] Nachbar F, Stolz W, Merkle T, Cognetta AB, Vogt T, Landthaler M, et al. The abcd rule of dermatoscopy: high prospective value in the diagnosis of doubtful melanocytic skin lesions. J Am Acad Dermatol 1994;30:551-9.

[19] Mariam, A.Sheha,Mai, S.Mabrouk, Amr Sharawy, "Automatic Detection of Melanoma Skin Cancer using Texture Analysis",

International Journal of Computer Applications, Volume 42, 2012.

[20] S. Li, J.T. Kwok, H. Zhu, Y. Wang, Texture classification using the support vector machines, Pattern Recognition 36 (12) (2003) 2883-2893. 
[21] A. Sengur, Wavelets transform and adaptive neuro-fuzzy inference system for color texture classification, Expert Systems with Applications 34 (3) (2008) 2120-2128.

[22] G.M. Haley, B.S. Manjunath, Rotation-invariant texture classification using a complete space-frequency model, IEEE Transactions on Image Processing 8 (2) (1999) 225-269.

[23] H. Zhou, R. Wang, C. Wang, A novel extended local binary pattern operator for texture analysis, Information Sciences 178 (22) (2008) 4314-4325.

[24] W.T. Freeman, E.H. Adelsonk, The design and use of steerable filters, IEEE Transactions on Pattern Analysisand Machine Intelligence 13 (9) (1991) 891906.

[25] 25. E.P. Simoncelli, W.T. Freeman, E.H. Adeslon, D.J. Heeger, Shiftable multi-scale transforms, IEEE Transactions on Information Theory 38 (2) (1992) $587-$ 607.

[26] Jeffrey Glaister*, Student Member, IEEE, Robert Amelard, Student Member, IEEE, Alexander Wong, Member, IEEE, and David A. Clausi, Senior Member, IEEE" MSIM: Multistage Illumination Modeling of Dermatological Photographs for Illumination-Corrected Skin Lesion Analysis" IEEE TRANSACTIONS ON BIOMEDICAL ENGINEERING, VOL. 60, NO. 7, JULY 20131873
[27] José Fernández Alcón, C`alina Ciuhu, Warner ten Kate, Adrienne Heinrich, Natallia Uzunbajakava, Gertruud Krekels, Denny Siem, and Gerard de Haan" Automatic Imaging System With Decision Support for Inspection of Pigmented Skin Lesions and Melanoma Diagnosis"IEEE journal of selected topics in signal processing, vol. 3, no. 1 , february 2009

[28] JeffreyGlaister" Segmentation of Skin Lesions From Digital Images Using Joint Statistical Texture Distinctiveness" IEEE TRANSACTIONS ON BIOMEDICAL ENGINEERING, VOL. 61, NO. 4, APRIL 2014.

[29] J.H. Morra, Z. Tu, L.G. Apostolova, A.E. Green, A.W Toga, et al., Comparison of AdaBoost and support vector machines for detecting Alzheimer's disease through automated Hippocampal segmentation, IEEE Transactions on Med- ical Imaging 29 (1) (2010) 30-43.

[30] E. Song, D. Huang, G. Maa, C.-C. Hung, Semisupervised multi-class Adaboost by exploiting unlabeled data, Expert Systems with Applications 38 (6) (2011) 6720-600726.

[31] Z.-H. Zhou, M.-L. Zhang, Multi-instance multi-label learning with application to scene classification, in: Proceedings of the Advances in Neural Information Processing Systems (NIPS), Vancouver, Canada, 2006, pp.1609-1616. 